\title{
Integer points of entire functions
}

\author{
J.K. Langley
}

April 27, 2005

\begin{abstract}
A result is proved which implies the following conjecture of Osgood and Yang from 1976: if $f$ and $g$ are non-constant entire functions, such that $T(r, f)=O(T(r, g))$ as $r \rightarrow \infty$ and such that $g(z) \in \mathbb{Z}$ implies $f(z) \in \mathbb{Z}$, then there exists a polynomial $G$ with coefficients in $\mathbb{Q}$, such that $G(\mathbb{Z}) \subseteq \mathbb{Z}$ and $f=G \circ g$.

Keywords: entire functions, forward differences, Wiman-Valiron theory.

MSC 2000: 30D20, 30D35.
\end{abstract}

\section{Introduction}

This paper will use standard notation from Nevanlinna theory [5], including $T(r, g)$ for the Nevanlinna characteristic of a function $g$ meromorphic in the plane and, for an entire function $f$, $M(r, f)=\max \{|f(z)|:|z|=r\}$. The starting point of the paper is the following conjecture advanced by Osgood and Yang in [11].

Conjecture 1.1 Let $f$ and $g$ be non-constant entire functions, such that $T(r, f)=O(T(r, g))$ as $r \rightarrow \infty$, and such that $g(z) \in \mathbb{Z}$ implies $f(z) \in \mathbb{Z}$. Then there exists a polynomial $G$ with coefficients in $\mathbb{Q}$, such that $G(\mathbb{Z}) \subseteq \mathbb{Z}$ and $f=G \circ g$.

This question arose in [11] from the problem of determining when

$$
L=\frac{e^{2 \pi i f}-1}{e^{2 \pi i g}-1}
$$


is entire. Conjecture 1.1 was proved in [11] in the special case where $f$ and $g$ are both polynomials. For the corresponding result in which $f$ and $g$ are rational functions, see [1] and [4] (see also [3, Lemma 3] and [7, Lemma 5]).

Integer points of entire functions have been considered by many authors going back at least to Pólya, who proved [12, p.55] that an entire function $f$ with $M(r, f)=o\left(2^{r}\right)$ and taking integer values on $\mathbb{N}$ reduces to a polynomial. An analogous result for analytic functions of polynomial growth in a half-plane was established in [7, Lemma 5], and some applications to value distribution and differential equations appear in $[7,8,9]$. Results concerning entire functions taking integer values on subsets of $\mathbb{N}$, in terms of growth and densities, may be found in [2].

The results of the present paper will establish Conjecture 1.1 and rather more. In order to deduce that $f$ is a function of $g$ it turns out that a relatively mild growth condition suffices, and it is only necessary that $f(z) \in \mathbb{Z}$ whenever $g(z) \in \mathbb{N}$ and $z$ lies in certain regions in which $g(z)$ is approximated by a suitable monomial. We will prove the following theorem.

Theorem 1.1 Let $K>1$ and $\eta>0$. Let $f, g$ be meromorphic with finitely many poles in the plane, such that $g$ has a pole or essential singularity at $\infty$. Suppose that there exists an unbounded set $J \subseteq[1, \infty)$ with the following properties. For each $r \in J$ there exist $z_{1}$ and a positive integer $N=N_{r}$ such that:

$$
\begin{gathered}
\left|z_{1}\right|=r \quad \text { and } \quad g\left(z_{1}\right) \in(0, \infty) \\
g(z)=g\left(z_{1}\right)\left(z / z_{1}\right)^{N}(1+o(1)) \quad \text { for all } z \text { satisfying } \quad\left|\log \left(z / z_{1}\right)\right| \leq K \pi N^{-1} \\
N \log ^{+} M(r, f) \log M(r, g)=o\left(g\left(z_{1}\right)\right) ; \\
f(z) \in \mathbb{Z} \quad \text { for all } z \text { in }\left\{u \in \mathbb{C}: g(u) \in \mathbb{N},|u|<r,\left|\log \left(u / z_{1}\right)\right| \leq 10 \eta N^{-1}\right\}
\end{gathered}
$$

Then there exists an entire function $G$ satisfying $f=G \circ g$ and such that

$$
G(\mathbb{Z}) \subseteq \mathbb{Z} \quad \text { and } \quad \liminf _{R \rightarrow \infty} \frac{\log M(R, G)}{R}=0 .
$$

The proof of Theorem 1.1 depends on applying the forward difference method to obtain local approximations for $f$ in terms of $g$ (see $\S 2$ ). The key idea is then to use harmonic measure to extend the domain of approximation. Clearly, if $f, g$ and $G$ are as in Theorem 1.1 and $g$ has a pole in $\mathbb{C}$ then $G$ must be a polynomial. 
Theorem 1.1 may be applied whenever $g$ is locally approximated by a multiple of a power of $z$ as in (3) and (4): this is the case, for example, for $g(z)=e^{-z}+z^{4}$ near the positive real axis. The fact that every entire function $g$ has regions as in (3) and (4) follows from WimanValiron theory [6], from which some standard facts are summarized in the following lemma. To establish the notation for this lemma let $g$ be a non-constant entire function with Taylor series $g(z)=\sum_{n=0}^{\infty} a_{n} z^{n}$. Then for $r>0$ the central index $\nu(r, g)$ is the largest $n$ for which $\left|a_{n}\right| r^{n}=\max \left\{\left|a_{m}\right| r^{m}: m \geq 0\right\}$. The results of Lemma 1.1 are normally stated for transcendental entire functions, but obviously hold if $g$ is a non-constant polynomial, in which case $\nu(r, g)$ for large $r$ is the degree of $g$.

Lemma 1.1 ([6]) Let $g$ be a non-constant entire function, and let $0<\delta<1$. There exists a set $E \subseteq[1, \infty)$ of finite logarithmic measure with the following properties. For $r \in[1, \infty) \backslash E$ the central index $\nu(r, g)$ satisfies

$$
\nu(r, g) \leq(\log M(r, g))^{1+\delta} .
$$

Further, there exists $z_{1}$ with

$$
\left|z_{1}\right|=r \quad \text { and } \quad g\left(z_{1}\right)=\left|g\left(z_{1}\right)\right| \geq(1-o(1)) M(r, g)
$$

such that, with $N=\nu(r, g)$,

$$
M(s, g)=(s / r)^{N} M(r, g)(1+o(1)) \quad \text { for all s such that }|\log (s / r)| \leq N^{-2 / 3}
$$

and

$$
g(z)=g\left(z_{1}\right)\left(z / z_{1}\right)^{N}(1+o(1)) \quad \text { and } \quad|g(z)| \geq(1-o(1)) M(|z|, g)
$$

for all $z$ such that $\left|\log \left(z / z_{1}\right)\right| \leq N^{-2 / 3}$.

Lemma 1.1 is completely standard, apart from the condition that $g\left(z_{1}\right)$ is real and positive. However, for $r$ in $[1, \infty) \backslash E$ and $z^{*}$ with $\left|z^{*}\right|=r,\left|g\left(z^{*}\right)\right|=M(r, g)$, the results of [6] give (9), as well as

$$
g(z)=g\left(z^{*}\right)\left(z / z^{*}\right)^{N}(1+o(1)) \quad \text { for } \quad\left|\log \left(z / z^{*}\right)\right| \leq N^{-5 / 8} .
$$

Choosing $z_{1}$ with $\left|z_{1}\right|=r$ and $\arg \left(z_{1} / z^{*}\right)=O(1 / N)$ and $g\left(z_{1}\right) \in(0, \infty)$ gives (8) and $(10)$. 
Theorem 1.2 Let $0<\delta<1$ and $\eta>0$. Let $f, g$ be non-constant entire functions and suppose that there exists a set $H \subseteq[1, \infty)$, of infinite logarithmic measure, such that for each $r \in H$ there exists $z_{1}$ satisfying (8) and (10), with $N=\nu(r, g)$, and such that (5) holds. Assume further that

$$
\log M(r, f)=o\left(\frac{M(r, g)}{(\log M(r, g))^{2+\delta}}\right) \quad \text { for all } r \text { in } H
$$

Then there exists an entire function $G$ satisfying $f=G \circ g$ and (6).

If $(11)$ is replaced by

$$
\limsup _{r \rightarrow \infty, r \in H} \frac{T(r, f)}{T(r, g)}<\infty
$$

then there exists a polynomial $G$ with coefficients in $\mathbb{Q}$, such that $G(\mathbb{Z}) \subseteq \mathbb{Z}$ and $f=G \circ g$.

Conjecture 1.1 now follows from Theorem 1.2, since Lemma 1.1 shows that to every nonconstant entire function $g$ correspond $H, z_{1}$ and $N$ as in (8) and (10).

An example illustrating the sharpness of Theorem 1.2 is given in $\S 5$. The obvious choice $f(z)=z \sin (\pi g(z))$ shows that the hypothesis (11) cannot be deleted in Theorem 1.2.

\section{Application of the forward difference method}

Define the forward differences in the standard way [12, p.52] for $n \in \mathbb{N}$ by

$$
\Delta^{0} h(a)=h(a), \quad \Delta h(a)=\Delta^{1} h(a)=h(a+1)-h(a), \quad \Delta^{n+1} h(a)=\Delta^{n} h(a+1)-\Delta^{n} h(a) .
$$

It is easy to prove by induction that if $h(a), \ldots, h(a+n)$ are integers then so is $\Delta^{n} h(a)$. For $x \in \mathbb{R}$ denote by $[x]$ the greatest integer not exceeding $x$.

Lemma 2.1 Let the real numbers $\eta, \varepsilon, S$ and $R$ satisfy the five conditions:

$$
0<\eta<\frac{1}{4} ; \quad 0<\varepsilon<\frac{\eta}{48} ; \quad 12 e^{S} 3^{-S}<1 ; \quad 1 \leq S<\varepsilon R ; \quad R \in \mathbb{N} .
$$

Let $F$ be an analytic function in the region

$$
\Sigma=\{w \in \mathbb{C}:|\log (w / R)|<2 \eta\}
$$

that satisfies

$$
F(\Sigma \cap \mathbb{N}) \subseteq \mathbb{Z} \quad \text { and } \quad \log |F(w)| \leq S \quad \text { for all } w \text { in } \quad \Sigma
$$


Then there exists a polynomial

$$
P_{q}(w)=P(w)=F(R)+(w-R) \Delta F(R)+\ldots+\frac{(w-R) \ldots(w-R-q+1)}{q !} \Delta^{q} F(R)
$$

of degree at most $q=[S]$, with coefficients satisfying

$$
\left|\frac{\Delta^{m} F(R)}{m !}\right| \leq 6 e^{S}
$$

for $0 \leq m \leq q$, such that $P(\mathbb{Z}) \subseteq \mathbb{Z}$ and

$$
|F(w)-P(w)|<12 e^{S} 3^{-\varepsilon R}
$$

for all $w$ in the region

$$
(1-\varepsilon) R<|w|<(1+\varepsilon) R, \quad|\arg w|<\varepsilon
$$

The proof of Lemma 2.1 requires a number of intermediate lemmas. Throughout this section, $F$ is the function of Lemma 2.1 .

Lemma 2.2 Let $C$ be the contour describing once counter-clockwise the boundary of the region

$$
|\log (z / R)| \leq \eta
$$

where $R$ and $\eta$ are as in Lemma 2.1, and suppose that $w$ lies inside $C$, and that $n \in \mathbb{N}$ with $R+n<R e^{\eta}$. Then

$$
F(w)-P_{n}(w)=\frac{1}{2 \pi i} \int_{C} \frac{(w-R) \ldots(w-R-n) F(t)}{(t-R) \ldots(t-R-n)(t-w)} d t,
$$

where $P_{n}(w)$ is a polynomial of degree at most $n$, which equals $F(w)$ at the $n+1$ points $R, R+1, \ldots, R+n$, and is given by

$$
P_{n}(w)=F(R)+(w-R) \Delta F(R)+\ldots+\frac{(w-R) \ldots(w-R-n+1)}{n !} \Delta^{n} F(R) .
$$

Moreover, the polynomial $P_{n}$ maps $\mathbb{Z}$ into $\mathbb{Z}$, and the coefficients in (21) satisfy (17) for $0 \leq$ $m \leq \varepsilon R$.

Proof. (20) and (21) are completely standard: see, for example, [12, pp.52-53]. Since $R$ and the forward differences $\Delta^{j} F(R), j=0, \ldots, n$, are integers, by (13) and (15), and since

$$
\frac{p(p-1) \ldots(p-m+1)}{m !} \in \mathbb{Z} \quad \text { for } \quad p \in \mathbb{Z} \quad \text { and } \quad m \in \mathbb{N},
$$


the inclusion $P_{n}(\mathbb{Z}) \subseteq \mathbb{Z}$ follows.

It remains to prove that the coefficients satisfy (17) for $0 \leq m \leq \varepsilon R$. The $m$ 'th forward difference is given by [12, pp.52-53]

$$
\frac{\Delta^{m} F(R)}{m !}=\frac{1}{2 \pi i} \int_{C} \frac{F(t)}{(t-R) \ldots(t-R-m)} d t
$$

in which $\log |F(t)| \leq S$ by (15). Now (13) gives

$$
\left|e^{u}-1\right| \geq \eta-\eta^{2}-\eta^{3}-\ldots \geq \eta-2 \eta^{2}>\eta / 2 \quad \text { for } \quad|u|=\eta
$$

and the the image of the circle $|u|=\eta$ under $e^{u}$ has length at most $2 \pi \eta e<6 \pi \eta$. Thus

$$
\operatorname{dist}(C, R)>\eta R / 2>24 \varepsilon R>24 \text { and } \operatorname{length}(C) \leq 6 \pi R \eta \text {. }
$$

Hence $|t-R|>\frac{1}{2} \eta R$ and $|t-R-j|>\frac{1}{4} \eta R>1$ for $1 \leq j \leq \varepsilon R$ and $t \in C$, which gives (17).

Lemma 2.3 Let $w$ lie in the region (19), and let the contour $C$ and the polynomial $P_{n}$ be as in Lemma 2.2. Then $w$ lies inside $C$ and, with $\varepsilon$ as in (13),

$$
\left|F(w)-P_{n}(w)\right|<12 e^{S} 3^{-n-1} \quad \text { for } \quad n \leq \varepsilon R .
$$

Proof. Let $t$ lie on $C$. Then (13), (19) and (23) give

$$
|w-R|<3 \varepsilon R<\operatorname{dist}(C, R) \quad \text { and } \quad|t-w| \geq \frac{1}{2} \eta R-3 \varepsilon R \geq \frac{1}{4} \eta R .
$$

Further, if $j$ is an integer with $0 \leq j \leq \varepsilon R$, then

$$
\left|\frac{w-R-j}{t-R-j}\right| \leq \frac{4 \varepsilon R}{\eta R / 2-\varepsilon R}<\frac{4 \varepsilon R}{\eta R / 4}=\frac{16 \varepsilon}{\eta}<\frac{1}{3},
$$

and the lemma follows using (15), (20) and (23) again.

Lemma 2.4 Let $R, S$ and $\varepsilon$ be as in (13), and let the polynomial $P_{n}$ be as in Lemma 2.2.

(i) If $[S] \leq n \leq \varepsilon R$ then $F(R-1)=P_{n}(R-1)$.

(ii) If $[S]+1 \leq n \leq \varepsilon R$ then $\Delta^{n} F(R)=0$. 
Proof. The point $w=R-1$ lies in the region (19), by (13). Hence Lemma 2.3 gives, for $[S] \leq n \leq \varepsilon R$,

$$
\left|F(R-1)-P_{n}(R-1)\right|<12 e^{S} 3^{-n-1}<12 e^{S} 3^{-S}<1,
$$

using (13) again. Part (i) is thus proved, since $R-1$ is an integer by (13), and $F(R-1)$ and $P_{n}(R-1)$ are both integers by $(15)$ and Lemma 2.2 .

Part (ii) now follows using part (i), since for $[S]+1 \leq n \leq \varepsilon R$,

$$
F(R-1)=P_{n-1}(R-1)=P_{n}(R-1)=P_{n-1}(R-1)+(-1)^{n} \Delta^{n} F(R) .
$$

To complete the proof of Lemma 2.1, let $w$ lie in the region (19), and let $R, S$ and $\varepsilon$ be as in (13). Let $P=P_{q}$ be given by (16), with $q=[S]$, and let $n=[\varepsilon R]$. Then $q \leq n$ by (13), and (17) follows from Lemma 2.2. Finally, Lemma 2.3 and part (ii) of Lemma 2.4 give

$$
|F(w)-P(w)|=\left|F(w)-P_{q}(w)\right|=\left|F(w)-P_{n}(w)\right|<12 e^{S} 3^{-n-1}<12 e^{S} 3^{-\varepsilon R} .
$$

\section{$3 \quad$ Proof of Theorem 1.1}

Let $f, g$ be as in the hypotheses, and assume without loss of generality that $\eta$ is small. If $f$ is a rational function with $f(\infty)$ finite then (2), (3) and (5) show that $f$ takes a fixed integer value $n_{0}$ infinitely often and so is constant. Assume henceforth that $f$ either is transcendental or has a pole at $\infty$. Choose $R_{0} \in(2, \infty)$, so large that

$$
f^{-1}(\{\infty\}) \cup g^{-1}(\{\infty\}) \subseteq B\left(0, \frac{1}{2} R_{0}\right),
$$

where $B(a, r)=\{z \in \mathbb{C}:|z-a|<r\}$. Then the function

$$
v(z)= \begin{cases}M\left(R_{0}, g\right), & \text { if }|z| \leq R_{0}, \\ \max \left\{|g(z)|, M\left(R_{0}, g\right)\right\}, & \text { if }|z|>R_{0},\end{cases}
$$

is subharmonic in the plane, and

$$
M(r, g)=\max \{|g(z)|:|z|=r\}=\max \left\{|g(z)|: R_{0} \leq|z| \leq r\right\}
$$

for large $r$. Similar considerations apply to $M(r, f)$. 
Lemma 3.1 With the notation of Theorem 1.1 , let $r \in J$ be large. Then there exists $z_{0}$ satisfying

$$
\left|z_{0}\right|=r^{\prime}<r \quad \text { and } \quad-5 \eta N^{-1}<\log \left|z_{0} / z_{1}\right|<-4 \eta N^{-1} \quad \text { and } \quad\left|\arg \left(z_{0} / z_{1}\right)\right| \leq \eta N^{-1} \text {, }
$$

such that

$$
R=g\left(z_{0}\right) \in \mathbb{N} \quad \text { and } \quad g\left(z_{0}\right) \geq(1-o(1)) e^{-5 \eta} g\left(z_{1}\right) \geq \frac{1}{2} g\left(z_{1}\right)
$$

and such that

$$
g(z)=g\left(z_{0}\right)\left(z / z_{0}\right)^{N}(1+o(1)) \text { for all } z \text { such that }\left|\log \left(z / z_{0}\right)\right| \leq 4 \eta N^{-1}
$$

Moreover, there exists a simply connected domain $\Omega=\Omega_{r}$ mapped univalently by $g$ onto the region $\Sigma$ defined by (14), and

$$
\Omega \subseteq\left\{z \in \mathbb{C}:\left|\log \left(z / z_{0}\right)\right| \leq 4 \eta N^{-1}\right\} \subseteq B(0, r)
$$

Finally

$$
S=\log M(r, f)=o(R)
$$

and on $\Omega$ the function $f$ may be written in the form $f(z)=F(g(z))$ with $F$ analytic on $g(\Omega)=\Sigma$ and satisfying (15).

Proof. The existence of $z_{0}$ satisfying (26), (27) and (28) follows at once from (2) and (3) since $\eta$ is small, and (28) in turn implies the existence of $\Omega$ satisfying (29). The function $F$ is defined on $g(\Omega)$ by $F=f \circ g^{-1}$, and (15), with $S=\log M(r, f)$, follows from (5), the remark following (25), (26) and (29). Finally, (30) follows from (4) and (27).

Let $\varepsilon$ be positive, but small compared to $\eta$, and use $c$ to denote a positive constant, not necessarily the same at each occurrence, but not depending on $\varepsilon, \eta, K, r, f$ or $g$. For large $r \in J$ Lemma 2.1 may then be applied, since the conditions (13) are satisfied, using (27) and (30).

Lemma 3.2 Let $J$ be as in the hypotheses of Theorem 1.1 and let $P_{q}=P$ be as in Lemma 2.1. If $r \in J$ is sufficiently large then there exists an arc $A_{r}$ of the circle $|z|=r^{\prime}$, where $r^{\prime}$ is given by (26), such that $A_{r}$ has angular measure at least $c \varepsilon N^{-1}$ and

$$
\left|f(z)-P_{q}(g(z))\right|<\exp (-c \varepsilon R) \quad \text { for all } z \text { in } A_{r} .
$$


Further, with $R_{0}$ as in (24),

$$
\left|P_{q}(g(z))\right| \leq c S e^{S}(3 M(r, g))^{S} \quad \text { for } \quad R_{0} \leq|z| \leq r^{\prime}
$$

Proof. The estimate (31) follows at once from (18) and (30), since if the positive constant $c$ is chosen small enough the image under $g$ of the arc $z=z_{0} e^{i \theta},-c \varepsilon N^{-1} \leq \theta \leq c \varepsilon N^{-1}$, lies in the region (19), by (28). Next, if $w=g(z)$, with $R_{0} \leq|z| \leq r^{\prime}$ and $j=0,1, \ldots, q$, then

$$
|w-R-j| \leq M(r, g)+R+S \leq M(r, g)+R(1+o(1)) \leq 3 M(r, g)
$$

using (25), (26), (27) and (30). Thus (32) follows from (16) and (17).

Lemma 3.3 Let $\sigma>0$. Then, with the hypotheses of Lemma 3.2, if $r \in J$ is sufficiently large,

$$
\max \left\{\left|f(z)-P_{q}(g(z))\right|: R_{0} \leq|z| \leq r / 2\right\}<\sigma .
$$

Proof. By (16) and (24) there exist $c>0$ and a monic polynomial $h_{q}$, with degree at most $c q \leq c S$ and with all its zeros in $|z|<\frac{1}{2} R_{0}$, such that the function

$$
u(z)=\log \left|h_{q}(z)\left(f(z)-P_{q}(g(z))\right)\right|
$$

is subharmonic on the disc $B\left(0, r^{\prime}\right)$. Then, for $z \in B\left(0, r^{\prime}\right)$,

$$
u(z) \leq c S \log M(r, g)+2 c S \log r \leq c \log M(r, f) \log M(r, g)=o\left(N^{-1} R\right)
$$

by (4), (27), (30), (32) and the maximum principle. On the other hand, (4), (27) and (31) give

$$
u(z)<-c \varepsilon R+2 c S \log r<-c \varepsilon R \quad \text { for } z \text { in } A_{r},
$$

in which $A_{r}$ is the arc of Lemma 3.2. Next, if $|z| \leq r / 2$ then the harmonic measure of the arc $A_{r}$ with respect to $B\left(0, r^{\prime}\right)$, evaluated at $z$, satisfies $\omega\left(z, A_{r}, B\left(0, r^{\prime}\right)\right) \geq c \varepsilon N^{-1}$ by Poisson's formula. Applying the two-constants theorem [10, p.42] to $u$ now gives

$$
u(z) \leq o\left(N^{-1} R\right)-c \varepsilon^{2} N^{-1} R<-c \varepsilon^{2} N^{-1} R \quad \text { for } \quad|z| \leq r / 2 .
$$

But $\left|h_{q}(z)\right| \geq 1$ for $|z| \geq R_{0}$, since $R_{0}>2$, and (33) follows using (4) and (27), provided $r$ is sufficiently large. 
Choose a sequence $r_{n} \rightarrow \infty, r_{n} \in J$. Then (33) gives polynomials $G_{n}$, each satisfying $G_{n}(\mathbb{Z}) \subseteq \mathbb{Z}$, such that

$$
\max \left\{\left|f(z)-G_{n}(g(z))\right|: R_{0} \leq|z| \leq s_{n}=r_{n} / 2\right\}=o(1) \quad \text { as } \quad n \rightarrow \infty
$$

The proof of Theorem 1.1 is completed by the next lemma.

Lemma 3.4 The polynomials $G_{n}$ in (34) have a subsequence converging locally uniformly on $\mathbb{C}$ to an entire function $G$ which satisfies $f=G \circ g$ and (6).

Proof. Let $T$ be large, and choose $r \in J$ and $z_{1}$ satisfying (2), (3) and (4) with $g\left(z_{1}\right)>2 T$. Then (3) and the fact that $K>1$ show that $g(\zeta)$ assumes in $R_{0} \leq|\zeta|<r$ all values $w$ lying on a circle $|w|=T^{\prime} \geq g\left(z_{1}\right) / 2>T$. If $n$ is so large that $s_{n} \geq r$ then (34) gives

$M\left(T, G_{n}\right) \leq M\left(T^{\prime}, G_{n}\right) \leq \max \left\{\left|G_{n}(g(z))\right|: R_{0} \leq|z| \leq r\right\} \leq \max \left\{|f(z)|: R_{0} \leq|z| \leq r\right\}+1$.

Hence the $G_{n}$ form a normal family on $\mathbb{C}$. Assume without loss of generality that the sequence $G_{n}$ converges locally uniformly on $\mathbb{C}$ to an entire function $G$. Then $G(\mathbb{Z}) \subseteq \mathbb{Z}$, and $f=G \circ g$ by (34). Moreover, using the remark following (25),

$$
M\left(T^{\prime}, G\right) \leq \max \left\{|G(g(z))|: R_{0} \leq|z| \leq r\right\}=\max \left\{|f(z)|: R_{0} \leq|z| \leq r\right\}=M(r, f),
$$

and so (4) gives

$$
\log M\left(T^{\prime}, G\right) \leq \log M(r, f)=o\left(g\left(z_{1}\right)\right)=o\left(T^{\prime}\right)
$$

\section{Proof of Theorem 1.2}

Let $f, g, \delta, \eta$ and the set $H$ be as in the hypotheses. Assume without loss of generality that $H \cap E=\emptyset$, with $E$ the exceptional set of Lemma 1.1, and for large $r \in H$ choose $z_{1}$ as in the statement of the theorem. Then (7), (8), (10) and (11) show that (2), (3) and (4) are satisfied, with $N=\nu(r, g)$. Since $f$ satisfies (5) it follows at once from Theorem 1.1 that there exists an entire function $G$ satisfying $f=G \circ g$ and (6). This proves the first part of Theorem 1.2. 
Suppose next that (11) is replaced by (12). Applying the Borel lemma [5, p.38] in the standard way to $h(s)=T\left(e^{s}\right)$, where $T(r)=T(r, f)$, shows that

$$
T\left(r e^{1 / T(r)}\right)=O(T(r)) \quad \text { for } \quad r \in[1, \infty) \backslash E_{0},
$$

in which $E_{0}$ has finite logarithmic measure and may be assumed without loss of generality to have empty intersection with $H$. Now (12), (35) and standard estimates give, for $r \in H$,

$$
\log M(r, f)=O\left(T(r) T\left(r e^{1 / T(r)}\right)\right)=O(T(r))^{2}=O(T(r, g))^{2}=O(\log M(r, g))^{2}
$$

Thus $f$ satisfies (11) and so there exists an entire function $G$ with $G(\mathbb{Z}) \subseteq \mathbb{Z}$ and $f=G \circ g$. Then $G$ is a polynomial, by $(12)$ and $[5, p .54]$, and it is easy to see that $G$ has rational coefficients.

Corollary 4.1 Let $f$ and $g$ be non-constant entire functions satisfying (12), where $H$ has infinite logarithmic measure, and suppose that the function $L$ defined by $(1)$ is entire. Then there exists a polynomial $G$ with coefficients in $\mathbb{Q}$, such that $G(\mathbb{Z}) \subseteq \mathbb{Z}$ and $f=G \circ g$.

\section{An example}

It seems worth observing that the condition (6) does not force an entire function $G$ to be a polynomial. To see this, for $n \in \mathbb{N}$ let

$$
R_{n}(z)=\frac{z\left(z^{2}-1\right) \ldots\left(z^{2}-n^{2}\right)}{(2 n+1) !}=\frac{(z+n) \ldots z \ldots(z-n)}{(2 n+1) !} .
$$

Thus $R_{n}(\mathbb{Z}) \subseteq \mathbb{Z}$ by $(22)$. Next, let $\psi$ be a non-decreasing positive function on $[1, \infty)$ with $\lim _{s \rightarrow \infty} \psi(s)=\infty$ and $\psi(s) \leq s$, and choose integers $n_{k}, \mu_{k}, \lambda_{k}, k=1,2, \ldots$, such that

$$
1 \leq n_{k}<\psi\left(\mu_{k}\right) \leq \mu_{k}<\lambda_{k}^{o(1)}<\lambda_{k}<n_{k+1} \quad \text { and } \quad \lambda_{k}^{2 \lambda_{k}}<2^{n_{k+1}-\lambda_{k}}
$$

Since, for $n \in \mathbb{N}$,

$$
\left|\frac{z^{2}-n^{2}}{2 n(2 n+1)}\right| \leq \begin{cases}s^{2}, & \text { if } \max \{n,|z|\} \leq s \\ \frac{1}{2}, & \text { if }|z| \leq n\end{cases}
$$

it follows on writing

$$
\frac{R_{n_{k+1}}(z)}{R_{n_{k}}(z)}=\left(\frac{z^{2}-\left(n_{k}+1\right)^{2}}{\left(2 n_{k}+2\right)\left(2 n_{k}+3\right)}\right) \ldots\left(\frac{z^{2}-\lambda_{k}^{2}}{2 \lambda_{k}\left(2 \lambda_{k}+1\right)}\right) \ldots\left(\frac{z^{2}-n_{k+1}^{2}}{2 n_{k+1}\left(2 n_{k+1}+1\right)}\right)
$$


that, using (37),

$$
\left|\frac{R_{n_{k+1}}(z)}{R_{n_{k}}(z)}\right| \leq \lambda_{k}^{2\left(\lambda_{k}-n_{k}\right)} 2^{\lambda_{k}-n_{k+1}} \leq \lambda_{k}^{-2 n_{k}} \leq \frac{1}{2} \quad \text { for } \quad|z| \leq \lambda_{k} .
$$

Further,

$$
\left|R_{n}(z)\right| \leq \frac{s\left(2 s^{2}\right)^{n}}{(2 n+1) !} \leq \frac{(2 s)^{2 n+1}}{(2 n+1) !} \quad \text { for } \quad|z|=s \geq n \in \mathbb{N}
$$

Set

$$
G(z)=\sum_{k=1}^{\infty} R_{n_{k}}(z)
$$

Let

$$
m \in \mathbb{N} \quad \text { and } \quad \mu_{m} \leq|z|=s \leq \lambda_{m}
$$

Then (37), (38) and (39) give

$$
\sum_{k=1}^{\infty}\left|R_{n_{k}}(z)\right| \leq \sum_{k=1}^{m-1}\left|R_{n_{k}}(z)\right|+\left|R_{n_{m}}(z)\right|\left(1+\frac{1}{2}+\frac{1}{4}+\ldots\right) \leq 2 \sum_{k=1}^{m}\left|R_{n_{k}}(z)\right| \leq 2 e^{2 s} .
$$

In particular the series (40) converges locally uniformly in $\mathbb{C}$, and $G$ is entire with $G(\mathbb{Z}) \subseteq \mathbb{Z}$.

To prove that $G$ is transcendental, define $h_{m}$ and $G_{m}$ by

$$
h_{m}(z)=\sum_{k=m+1}^{\infty} R_{n_{k}}(z), \quad G(z)=G_{m}(z)+h_{m}(z) .
$$

Then $G_{m}(z)$ is a polynomial of degree $2 n_{m}+1$ with $G_{m}^{\left(2 n_{m}+1\right)}(z) \equiv 1$. Since $h_{m}(j)=0$ for $j=-n_{m}-1,-n_{m}, \ldots, n_{m}, n_{m}+1$ by (36), repeated application of Rolle's theorem shows that there exists $x_{m} \in\left(-n_{m}-1, n_{m}+1\right)$ with $h_{m}^{\left(2 n_{m}+1\right)}\left(x_{m}\right)=0$ and hence $G^{\left(2 n_{m}+1\right)}\left(x_{m}\right)=1$.

For $z$ satisfying (41), inequalities (37), (38), (39) and (42) imply that

$$
|G(z)| \leq 2 \sum_{k=1}^{m}\left|R_{n_{k}}(z)\right| \leq 2 m(2 s)^{2 n_{m}+1} \leq 2 n_{m}(2 s)^{2 n_{m}+1},
$$

which gives, for some $c>0$,

$$
\log M(s, G) \leq\left(2 n_{m}+1\right) \log 2 s+\log 2 n_{m} \leq c \psi(s) \log s \quad \text { for } \quad \mu_{m} \leq s \leq \lambda_{m} .
$$

Thus $G$ may have arbitrarily slow lower growth, subject to being transcendental, even on a set of upper logarithmic density 1.

Finally, the choice $g(z)=e^{z}, f(z)=G\left(e^{z}\right)$, together with (43), gives $T(r, f) \leq \log M(r, f) \leq \log M(M(r, g), G) \leq c \psi(M(r, g)) \log M(r, g) \leq c \pi \psi\left(e^{\pi T(r, g)}\right) T(r, g)$ for $r$ in the union $H$ of the intervals $\left[\log \mu_{m}, \log \lambda_{m}\right]$, and $H$ has infinite logarithmic measure, by (37). Since $\psi \circ$ exp can grow arbitrarily slowly, the second part of Theorem 1.2 is sharp. 


\section{Functions taking integer values at the same points}

The following result addresses the natural question of what may be deduced if two non-constant entire functions take integer values at the same points.

Theorem 6.1 Let $f$ and $g$ be non-constant entire functions with the following property. There exist $\varepsilon \in(0,1)$ and a set $H \subseteq[1, \infty)$ of infinite logarithmic measure such that for $r \in H$ :

(i) $f(\zeta) \in \mathbb{Z}$ for all $\zeta$ satisfying

$$
r e^{-\varepsilon / \nu(r, g)}<|\zeta|<r \quad \text { and } \quad g(\zeta) \in \mathbb{N} \quad \text { and } \quad|g(\zeta)|>(1-\varepsilon) M(|\zeta|, g)
$$

(ii) $g(\zeta) \in \mathbb{Z}$ for all $\zeta$ satisfying

$$
r e^{-\varepsilon / \nu(r, f)}<|\zeta|<r \quad \text { and } \quad f(\zeta) \in \mathbb{N} \quad \text { and } \quad|f(\zeta)|>(1-\varepsilon) M(|\zeta|, f)
$$

Then there exists $q \in \mathbb{Z}$ such that $f= \pm g+q$.

Proof. Let $F(z)=\sin (\pi f(z))$. Then

$$
T(r, F) \leq \log M(r, F) \leq c M(r, f)
$$

denoting by $c$ a positive constant, not necessarily the same at each occurrence. Applying the Borel lemma [5, p.38] gives (35), this time with $T(r)=T(r, F)$, in which the exceptional set $E_{0}$ has finite logarithmic measure. Without loss of generality both $E_{0}$ and the exceptional set $E$ of Lemma 1.1 may be assumed to have empty intersection with $H$. This gives

$$
n(r, 1 / F) \leq c T(r) N\left(r e^{1 / T(r)}, 1 / F\right) \leq c T(r, F)^{2} \quad \text { for } \quad r \in H .
$$

Let $\eta$ be small and positive, and for large $r \in H$ choose $z_{1}$ satisfying (8) and (10), with $N$ the central index of $g$. Then (5) is satisfied, and (9) and (10) show that there exist at least $c M(r, g)$ points $\zeta$ satisfying (44). On combination with (45) and (46) this leads to

$$
c M(r, g) \leq n(r, 1 / F) \leq c T(r, F)^{2} \leq c M(r, f)^{2}
$$

from which it follows, applying the same argument with $f$ and $g$ interchanged, that

$$
\log M(r, g) \leq c \log M(r, f) \leq c \log M(r, g) \quad \text { for } \quad r \in H
$$


Applying Theorem 1.2 twice now gives entire functions $G$ and $G_{1}$, each mapping $\mathbb{Z}$ into $\mathbb{Z}$, such that $f=G \circ g, g=G_{1} \circ f$. Here $G_{1}=G^{-1}$ and so $G$ is a linear function $G(\zeta)=p \zeta+q$. Since $G(0), G(1), G_{1}(0), G_{1}(1)$ are integers it follows that $q \in \mathbb{Z}$ and $p= \pm 1$.

The paper concludes with a simple result for meromorphic functions.

Theorem 6.2 Let $f$ and $g$ be functions meromorphic on a plane domain $U$, such that $g$ has a pole at $a \in U$ and $f(z) \in \mathbb{Z}$ whenever $z \in U$ and $g(z) \in \mathbb{N}$. Then $f$ is a polynomial in $g$.

Proof. Choose $V=B(a, r)$ such that $f$ has no poles in $0<|z-a| \leq r$. Then $w=g(z)$ maps a subdomain $W$ of $V$ conformally onto a half-plane $H=\{w \in \mathbb{C}: \operatorname{Re}(w)>M>1\}$. Set $f(z)=F(g(z))$ for $z \in W$. Then there exists $N>0$ such that $|F(w)| \leq|w|^{N}$ on $H$, and $F(w) \in \mathbb{Z}$ for $w \in H \cap \mathbb{N}$. By [7, Lemma 5], $F$ is a polynomial.

The author thanks Gary Gundersen for useful correspondence and for drawing his attention to references [1] and [4], and the referee for some very helpful suggestions to improve the readability of the paper.

\section{References}

[1] I.N. BAKER, Solution to Advanced Problem 6410, Amer. Math. Monthly 91, May 1984, 317.

[2] A.O. GELFOND, Transcendental and algebraic numbers (Dover, New York, 1960).

[3] G.G. GUNDERSEN, When two entire functions and also their first derivatives have the same zeros, Indiana Univ. Math. J. 30, No. 2 (1981), 293-303.

[4] G.G. GUNDERSEN and S. OSBORN, Advanced Problem 6410, Amer. Math. Monthly 89, Dec. 1982, 787-788.

[5] W.K. HAYMAN, Meromorphic functions (Clarendon Press, Oxford, 1964).

[6] W.K. HAYMAN, The local growth of power series: a survey of the Wiman-Valiron method, Canad. Math. Bull. 17 (1974), 317-358. 
[7] J.K. LANGLEY, On second order linear differential polynomials, Results Math. 26 (1994), 51-82.

[8] J.K. LANGLEY, Two results related to a question of Hinkkanen, Kodai Math. J. 19 (1996), $52-61$.

[9] J.K. LANGLEY, Quasiconformal modifications and Bank-Laine functions, Arch. Math. (Basel) 71 (1998), 233-239.

[10] R. NEVANLINNA, Eindeutige analytische Funktionen, 2. Auflage, Grundlehren Math. Wiss. 46 (Springer, Berlin, 1953).

[11] C.F. OSGOOD and C.C. YANG, On the quotient of two integral functions, J. Math. Anal. Appl. 54 (1976), 408-418.

[12] J.M. WHITTAKER, Interpolatory function theory, Cambridge Tract No. 33 (Cambridge University Press 1935).

J.K. Langley

School of Mathematical Sciences

University of Nottingham

Nottingham, NG7 2RD

jkl@maths.nott.ac.uk 